\title{
Fixed point theorems on a closed ball
}

\author{
Manish Chandra Singh, Mahesh Chandra Joshi, \\ NAVEEN CHANDRA
}

Abstract. The aim of the paper is to obtain some fixed point theorems for extended $(\varphi, F)$-weak type contraction on a closed ball in metric spaces. Our results generalize some recently established results.

\section{INTRODUCTION}

In 2012, Samet et al. [8] introduced a class of $\alpha$-admissible mapping.

Definition 1 ([8]). Let $T: X \rightarrow X$ and $\alpha: X \times X \rightarrow[0,+\infty)$. We say that $T$ is $\alpha$-admissible if $x, y \in X, \alpha(x, y) \geq 1$ implies that $\alpha(T x, T y) \geq 1$.

Definition 2 ([7]). Let $T: X \rightarrow X$ and $\alpha, \eta: X \times X \rightarrow[0,+\infty)$ be two functions. We say that $T$ is $\alpha$-admissible mapping with respect to $\eta$ if $x, y \in X, \alpha(x, y) \geq \eta(x, y)$ implies that $\alpha(T x, T y) \geq \eta(T x, T y)$.

If $\eta(x, y)=1$, then Definition 2 reduces to Definition 1 . If $\alpha(x, y)=1$, then $T$ is called an $\eta$-subadmissible mapping.

Definition $3([4])$. Let $(X, d)$ be a metric space. Let $T: X \rightarrow X$ and $\alpha, \eta: X \times X \rightarrow[0,+\infty)$ be two functions. We say that $T$ is $\alpha-\eta$-continuous mapping on $(X, d)$ if for given $x \in X$ and sequence $\left\{x_{n}\right\}$ with $x_{n} \rightarrow x$ as $n \rightarrow \infty ; \alpha\left(x_{n}, x_{n+1}\right) \geq \eta\left(x_{n}, x_{n+1}\right), \quad$ for all $n \in \mathbb{N} \Rightarrow T x_{n} \rightarrow T x$. Definition $4([6])$. Let $(X, d)$ be a metric space. A mapping $T: X \rightarrow X$ is said to be an $F$-contraction if there exists $\tau>0$ such that

$$
\forall x, y \in X, d(T x, T y)>0 \Rightarrow \tau+F(d(T x, T y)) \leq F(d(x, y)),
$$

where $F: \mathbb{R}_{+} \rightarrow \mathbb{R}$ is a mapping satisfying the following conditions:

$\left(\mathrm{F}_{1}\right) F$ is strictly increasing, i.e., for all $x, y \in \mathbb{R}_{+}$such that $x<y$, $F(x)<F(y)$

$\left(\mathrm{F}_{2}\right)$ For each sequence $\left\{\alpha_{n}\right\}_{n=1}^{\infty}$ of positive numbers, $\lim _{n \rightarrow \infty} \alpha_{n}=0$ if and only if $\lim _{n \rightarrow \infty} F\left(\alpha_{n}\right)=-\infty$;

2020 Mathematics Subject Classification. Primary: 47H10; Secondary: 54H25.

Key words and phrases. $\alpha$-admissible, $\alpha$ - $\eta$-continuous, $\alpha-\eta-G F$-contraction, $\quad F$ contraction, fixed point.

Full paper. Received 18 September 2020, revised 30 September 2020, accepted 15 October 2020, available online 16 March 2021. 
$\left(\mathrm{F}_{3}\right)$ There exists $k \in(0,1)$ such that $\lim _{\alpha \rightarrow 0^{+}} \alpha^{k} F(\alpha)=0$.

We denote by $\Delta_{F}$, the set of all functions satisfying the conditions $\left(\mathrm{F}_{1}\right)-\left(\mathrm{F}_{3}\right)$.

Wardowski [9] modified Banach contraction principle for $F$-contraction as follows.

Theorem 1. Let $(X, d)$ be a complete metric space and let $T: X \rightarrow X$ be an $F$-contraction. Then $T$ has a unique fixed point $z \in X$ and for every $x \in X$ the sequence $\left\{T^{n} x\right\}_{n \in \mathbb{N}}$ converges to $z$.

Hussain et al. [4] introduced the following family of new functions. Let $\Delta_{G}$ denote the set of all functions $G: \mathbb{R}_{+}^{4} \rightarrow \mathbb{R}_{+}$satisfying:

(G) for all $t_{1}, t_{2}, t_{3}, t_{4} \in \mathbb{R}_{+}$with $t_{1} t_{2} t_{3} t_{4}=0$, there exists $\tau>0$ such that $G\left(t_{1}, t_{2}, t_{3}, t_{4}\right)=\tau$.

Definition 5 ([4]). Let $(X, d)$ be a metric space and $T$ be a self-mapping on $X$. Also, suppose that $\alpha, \eta: X \times X \rightarrow[0,+\infty)$ are two function. We say that $T$ is $\alpha-\eta-G F$-contraction, if for $x, y \in X$ with $\eta(x, T x) \leq \alpha(x, y)$ and $d(T x, T y)>0$, we have

$$
G(d(x, T x), d(y, T y), d(x, T y), d(y, T x))+F(d(T x, T y)) \leq F(d(x, y)),
$$

where $G \in \Delta_{G}$ and $F \in \Delta_{F}$.

For $x \in X$ and $\epsilon>0, \overline{B(x, \epsilon)}=\{y \in X: d(x, y) \leq \epsilon\}$ is a closed ball in $(X, d)$. The following result, regarding the existence of the fixed point of the mapping satisfying a contractive condition on the closed ball, was given in [5]. The result is very useful in the sense that it requires the contraction condition only on a closed ball, instead of on the whole space.

Theorem 2 ([5]). Let $(X, d)$ be a complete metric space, $T: X \rightarrow X$ be a mapping, $r>0$ and $x_{0}$ be an arbitrary point in $X$. Suppose there exists $k \in[0,1)$ with

$$
d(T x, T y) \leq k d(x, y), \quad \text { for all } x, y \in Y=\overline{B\left(x_{0}, r\right)},
$$

and $d\left(x_{0}, T x_{0}\right)<(1-k) r$. Then there exists a unique point $x *$ in $\overline{B\left(x_{0}, r\right)}$ such that $x *=T x *$.

Recently, in 2019, Hussain [3] introduced the Ćirić type modified Fcontraction on a closed ball in a complete metric space.

Definition 6 ([3]). Let $(X, d)$ be a metric space. A self-mapping $T: X \rightarrow X$ is said to be a modified $F$-contraction via $\alpha$-admissible mappings if there exists $\tau>0$ such that

$$
d(T x, T y)>0 \Rightarrow \tau+F(\alpha(x, y) d(T x, T y)) \leq F(\psi(M(x ; y))),
$$

where

$$
M(x, y)=\max \left\{d(x, y), d(x, T x), d(y, T y), \frac{d(x, T y)+d(y, T x)}{2}\right\}
$$


for all $x, y \in \overline{B\left(x_{0}, r\right)} \subseteq X$; where $F: \mathbb{R}^{+} \rightarrow \mathbb{R}$ is a mapping satisfying $(F 1)-(F 3)$ and $\psi \in \Psi$.

In Definition $6, \Psi$ be the familiy of functions of self-mappings on $[0, \infty)$ satisfying:

(i) $\psi$ is nondecreasing.

(ii) $\Sigma_{n=1}^{\infty} \psi^{n}(t)<+\infty$, for each $t>0$.

Remark 1. If $\psi \in \Psi$, then $\psi(t)<t$ for all $t>0$.

Using Definition 6, Hussain [3] obtained the following result.

Theorem $3([3])$. Let $(X, d)$ be a complete metric space. Let $T: X \rightarrow X$ be a modified $F$-contraction via $\alpha$-admissible mappings and $x_{0}$ be an arbitrary point in $X$. Assume that

$$
x, y \in \overline{B\left(x_{0}, r\right)}, \quad \tau+F(\alpha(x, y) d(T x, T y)) \leq F(\psi(M(x, y))),
$$

where $\tau>0$. Moreover

$$
\sum_{j=0}^{N} d\left(x_{0}, T x_{0}\right) \leq r, \text { for all } j \in \mathbb{N} \text { and } r>0 .
$$

Suppose that the following assertions hold:

(i) $T$ is an $\alpha$-admissible mapping;

(ii) there exist a point $x_{0} \in X$ such that $\alpha\left(x_{0}, T x_{0}\right) \geq 1$;

(iii) $T$ is continuous.

Then there exist a point $x *$ in $\overline{B\left(x_{0}, r\right)}$ such that $T x *=x *$.

In this paper, we obtain some fixed point results which generalize the results of Dey et al [1], Dung and Hang [2] and Hussain [3] on a closed ball in a complete metric space.

\section{Main Results}

Now, we introduce the following definition.

Definition 7. Let $(X, d)$ be a metric space. A self-mapping $T: X \rightarrow X$ is said to be a modified $F$-contraction II via $\alpha$-admissible mappings if there exists $\tau>0$ such that

$$
d(T x, T y)>0 \Rightarrow \tau+F(\alpha(x, y) d(T x, T y)) \leq F(\psi(M(x, y)))
$$

where,

$$
\begin{gathered}
M(x, y)=\max \left\{d(x, y), d(x, T x), d(y, T y), \frac{d(x, T y)+d(y, T x)}{2},\right. \\
\left.\frac{d\left(T^{2} x, x\right)+d\left(T^{2} x, T y\right)}{2}, d\left(T^{2} x, T x\right), d\left(T^{2} x, y\right), d\left(T^{2} x, T y\right)\right\},
\end{gathered}
$$

for all $x, y \in \overline{B\left(x_{0}, r\right)} \subseteq X$, and $F: \mathbb{R}_{+} \rightarrow \mathbb{R}$ is a mapping satisfying $\left(\mathrm{F}_{1}\right)-\left(\mathrm{F}_{3}\right)$ and $\psi \in \Psi$, where $\Psi$ is defined as the same in Definition 6. 
Theorem 4. Let $(X, d)$ be a complete metric space and $T: X \rightarrow X a$ modified $F$-contraction II via $\alpha$-admissible mappings and $x_{0}$ be an arbitrary point in $X$. Assume that

$$
x, y \in \overline{B\left(x_{0}, r\right)}, \quad \tau+F(\alpha(x, y) d(T x, T y)) \leq F(\psi(M(x, y))),
$$

where $\tau>0$. Moreover,

$$
\Sigma_{j=0}^{N} d\left(x_{j}, T x_{j}\right) \leq r, \quad \forall j \in \mathbb{N} \text { and } r>0 .
$$

Suppose that the following assertions hold:

(i) $T$ is an $\alpha$-admissible mapping;

(ii) there exist a point $x_{0} \in X$ such that $\alpha\left(x_{0}, T x_{0}\right) \geq 1$;

(iii) $T$ is continuous.

Then there exist a point $x *$ in $\overline{B\left(x_{0}, r\right)}$ such that $T x *=x *$.

Proof. Due to assumption (ii), there exist a point $x_{0} \in X$ such that $\alpha\left(x_{0}, T x_{0}\right) \geq 1$. Now, we construct a sequence $\left\{x_{n}\right\}_{n \geq 0}$ in $X$ such that $x_{n+1}=T x_{n} .\left\{x_{n}\right\}$ is a non-increasing sequence. If we assume that $x_{n}=$ $x_{n+1}$ for some $n \geq 0$, then the proof is complete obviously. So, we assume that $x_{n} \neq x_{n+1}$ for all $n \geq 0$. Since $\alpha\left(x_{0}, x_{1}\right)=\alpha\left(x_{0}, T x_{0}\right) \geq 1$ and $T$ is $\alpha$-admissible, we have

$$
\alpha\left(x_{n}, x_{n+1}\right) \geq 1, \quad \forall n \geq 0 .
$$

Firstly, we show that $x_{n} \in \overline{B\left(x_{0}, r\right)}$ for all $n \in \mathbb{N}$. For this, consider $d\left(x_{0}, x_{1}\right)=d\left(x_{0}, T x_{0}\right) \leq r$. Thus $x_{1} \in \overline{B\left(x_{0}, r\right)}$. Suppose that $x_{2}, \ldots, x_{j} \in$ $\overline{B\left(x_{0}, r\right)}$ for some $j \in \mathbb{N}$, then from (4),

$$
\begin{array}{r}
F\left(\alpha\left(x_{j-1}, x_{j}\right) d\left(T x_{j-1}, T x_{j}\right)\right) \leq F\left(\psi\left(M\left(x_{j-1}, x_{j}\right)\right)-\tau\right. \\
\Rightarrow d\left(x_{j}, x_{j+1}\right)<\psi\left(\left(M\left(x_{j-1}, x_{j}\right)\right)\right)<M\left(x_{j-1}, x_{j}\right)
\end{array}
$$

where

$$
\begin{aligned}
M\left(x_{j-1}, x_{j}\right)= & \max \left\{d\left(x_{j-1}, x_{j}\right), d\left(x_{j-1}, x_{j}\right), d\left(x_{j}, x_{j+1}\right),\right. \\
& \frac{d\left(x_{j-1}, x_{j+1}\right)+d\left(x_{j}, x_{j}\right)}{2}, \frac{d\left(x_{j+1}, x_{j-1}\right)+d\left(x_{j+1}, x_{j+1}\right)}{2}, \\
& \left.d\left(x_{j+1}, x_{j}\right), d\left(x_{j+1}, x_{j}\right), d\left(x_{j+1}, x_{j+1}\right)\right\} \\
= & \max \left\{d\left(x_{j-1}, x_{j}\right), d\left(x_{j}, x_{j+1}\right\} .\right.
\end{aligned}
$$

Therefore, we have

$$
\begin{aligned}
F\left(d\left(x_{j}, x_{j+1}\right)\right. & \leq F\left(\alpha\left(x_{j-1}, x_{j}\right) d\left(T x_{j-1}, T x_{j}\right)\right) \\
& \leq F\left(\max \left\{d\left(x_{j-1}, x_{j}\right), d\left(x_{j}, x_{j+1}\right)\right\}\right)-\tau .
\end{aligned}
$$

If $\max \left\{d\left(x_{j-1}, x_{j}\right), d\left(x_{j}, x_{j+1}\right)\right\}=d\left(x_{j}, x_{j+1}\right)$, then

$$
\Rightarrow F\left(d\left(x_{j}, x_{j+1}\right) \leq F\left(d\left(x_{j}, x_{j+1}\right)\right)-\tau .\right.
$$


This gives $\tau \leq 0$, a contradiction. Hence, $\max \left\{d\left(x_{j-1}, x_{j}\right), d\left(x_{j}, x_{j+1}\right)\right\}=$ $d\left(x_{j-1}, x_{j}\right)$. Now,

$$
\begin{aligned}
d\left(x_{0}, x_{j+1}\right) & \leq d\left(x_{0}, x_{1}\right)+\ldots+d\left(x_{j}, x_{j+1}\right) \\
& =\Sigma_{j=0}^{N} d\left(x_{j}, T x_{j}\right) \leq r .
\end{aligned}
$$

Therefore, $x_{j+1} \in \overline{B\left(x_{0}, r\right)}$ for all $n \in \mathbb{N}$. Continuing this process, we get

$$
\begin{aligned}
F\left(d\left(x_{n}, x_{n+1}\right)\right) \leq & F\left(d\left(x_{n-1}, x_{n}\right)\right)-\tau \\
& =F\left(d\left(T x_{n-2}, T x_{n-1}\right)\right)-\tau \\
& \leq F\left(d\left(x_{n-2}, x_{n-1}\right)\right)-2 \tau \\
& \quad \vdots \\
& \leq F\left(d\left(x_{0}, x_{1}\right)\right)-n \tau .
\end{aligned}
$$

This implies

$$
F\left(d\left(x_{n}, x_{n+1}\right)\right) \leq F\left(d\left(x_{0}, x_{1}\right)\right)-n \tau .
$$

Taking limit we get, $\lim _{n \rightarrow \infty} F\left(d\left(x_{n}, x_{n+1}\right)\right)=-\infty$. So, we have

$$
d\left(x_{n}, x_{n+1}\right)=0 .
$$

From $(F 3)$, there exists $k \in(0,1)$ such that

$$
\lim _{n \rightarrow \infty}\left(d\left(x_{n}, x_{n+1}\right)\right)^{k} F\left(d\left(x_{n}, x_{n+1}\right)\right)=0 .
$$

From (6), for all $n \in N$, we obtain

$$
\begin{aligned}
\left(d\left(x_{n}, x_{n+1}\right)\right)^{k}\left(F\left(d\left(x_{n}, x_{n+1}\right)\right)-F\left(d\left(x_{0}, x_{1}\right)\right)\right) & \leq \\
-\left(d\left(x_{n}, x_{n+1}\right)\right)^{k} n \tau & \leq 0 .
\end{aligned}
$$

By using (7), (8) and letting $n \rightarrow \infty$ in (9), we have

$$
\lim _{n \rightarrow \infty}\left(n\left(d\left(x_{n}, x_{n+1}\right)\right)^{k}\right)=0 .
$$

We observe that from (10), there exist $n_{1} \in \mathbb{N}$ such that $n\left(d\left(x_{n}, x_{n+1}\right)\right)^{k} \leq 1$ for all $n \geq n_{1}$, we get

$$
d\left(x_{n}, x_{n+1}\right) \leq \frac{1}{n^{k}}, \quad \forall n \geq n_{1} .
$$

Now $m, n \in \mathbb{N}$ such that $m>n \geq n_{1}$. Then by triangle inequality and from (11), we have

$$
\begin{aligned}
d\left(x_{n}, x_{m}\right) & \leq d\left(x_{n}, x_{n+1}\right)+d\left(x_{n+1}, x_{n+2}\right)+\cdots+d\left(x_{m-1}, x_{m}\right) \\
& =\Sigma_{i=n}^{m-1} d\left(x_{i}, x_{i-1}\right) \\
& \leq \Sigma_{i=n}^{\infty} d\left(x_{i}, x_{i+1}\right) \\
& \leq \sum_{i=n}^{\infty} \frac{1}{i^{\frac{1}{k}}} .
\end{aligned}
$$


The series $\frac{1}{i \frac{1}{k}}$ is convergent. Taking the limit as $n \rightarrow \infty$, in (12), we have $\lim _{n, m \rightarrow \infty} d\left(x_{n}, x_{m}\right)=0$. Hence $x_{n}$ is a Cauchy sequence. Since, $X$ is a complete metric space there exists an $x * \in \overline{B\left(x_{0}, r\right)}$ such that $x_{n} \rightarrow x *$ as $n \rightarrow \infty$. $T$ is a continuous then $x_{n+1}=T x_{n} \rightarrow T x *$ as $n \rightarrow \infty$. That is, $x *=T x *$. Hence $x *$ is a fixed point of $T$.

Motivating by the paper [1], we introduce the following definition.

Definition 8. Let $(X, d)$ be a metric space. A self-mapping $T: X \rightarrow X$ is said to be a modified $F$-contraction III via $\alpha$-admissible mappings if there exists $\tau>0$ such that

$$
d(T x, T y)>0 \quad \Rightarrow \quad \tau+F(\alpha(x, y) d(T x, T y)) \leq F\left(\psi\left(M^{\prime}(x, y)\right)\right),
$$

where

$$
\begin{aligned}
M^{\prime}(x, y) & =\max \left\{d(x, y), \frac{d(x, T y)+d(y, T x)}{2}, \frac{d\left(T^{2} x, x\right)+d\left(T^{2} x, T y\right)}{2},\right. \\
& \left.d\left(T^{2} x, T x\right), d\left(T^{2} x, y\right), d(T x, y)+d(y, T y), d\left(T^{2} x, T y\right)+d(x, T x)\right\},
\end{aligned}
$$

for all $x, y \in \overline{B\left(x_{0}, r\right)} \subseteq X$, and $F: \mathbb{R}_{+} \rightarrow \mathbb{R}$ is a mapping satisfying $\left(F_{1}\right)-\left(F_{3}\right)$ and $\psi \in \Psi$, where $\Psi$ is defined as the same in Definition 6 .

Remark 2. Every modified $F$-contraction III is a modified $F$-contraction II via $\alpha$-admissible mapping. The reverse implications do not hold.

Now we obtain the following result which is a generalization of Theorem 4 .

Theorem 5. Let $(X, d)$ be a complete metric space and $T: X \rightarrow X$ a modified $F$-contraction III via $\alpha$-admissible mappings and $x_{0}$ be an arbitrary point in $X$. Assume that

$$
x, y \in \overline{B\left(x_{0}, r\right)}, \quad \tau+F(\alpha(x, y) d(T x, T y)) \leq F\left(\psi\left(M^{\prime}(x, y)\right)\right),
$$

where $\tau>0$. Moreover,

$$
\Sigma_{j=0}^{N} d\left(x_{j}, T x_{j}\right) \leq r, \quad \forall j \in \mathbb{N} \text { and } r>0 .
$$

Suppose that the following assertions hold:

(i) $T$ is an $\alpha$-admissible mapping;

(ii) there exist a point $x_{0} \in X$ such that $\alpha\left(x_{0}, T x_{0}\right) \geq 1$;

(iii) $T$ is continuous.

Then there exist a point $x *$ in $\overline{B\left(x_{0}, r\right)}$ such that $T x *=x *$.

Proof. The proof is same as in Theorem 4.

Remark 3. Theorem 4 and 5 generalize the main result of Hussain [3] and also extends results of [2] and [1] on closed ball in a complete metric space. 


\section{Fixed Point Theorems for GF-Contraction on Closed Ball}

Definition 9. Let $T$ be a self mapping in a metric space $(X, d)$ and let $x_{0}$ be an arbitrary point in $X$. Also suppose that $\alpha: X \times X \rightarrow-\infty \cup$ $(0,+\infty) ; \eta: X \times X \rightarrow \mathbb{R}_{+}$are two functions. We say that $T$ is called modified $\alpha-\eta-\psi$-GF-contraction II on closed ball if for all $x, y \in \overline{B\left(x_{0}, r\right)} \subseteq X$; with $\eta(x, T x) \leq \alpha(x, y)$ and $d(T x, T y)>0$; we have

$$
\begin{gathered}
G(d(x, T x), d(y, T y), d(x, T y), d(y, T x))+F(d(T x, T y)) \leq \\
\leq F(\psi(M(x, y))),
\end{gathered}
$$

where

$$
\begin{aligned}
M(x, y)= & \max \left\{d(x, y), d(x, T x), d(y, T y), \frac{d(x, T y)+d(y, T x)}{2},\right. \\
& \left.\frac{d\left(T^{2} x, x\right)+d\left(T^{2} x, T y\right)}{2}, d\left(T^{2} x, T x\right), d\left(T^{2} x, y\right), d\left(T^{2} x, T y\right)\right\} .
\end{aligned}
$$

Moreover,

$$
\sum_{j=0}^{N} d\left(x_{j}, T x_{j}\right) \leq r, \quad \forall j \in \mathbb{N} \text { and } r>0,
$$

$G \in \Delta_{G}, \psi \in \Psi$, and $F \in \Delta_{F}$.

Theorem 6. Let $(X, d)$ be a complete metric space. Let $T: X \rightarrow X$ be an $\alpha-\eta-\psi-G F$-contraction II mapping on closed ball satisfying the following assertions:

(i) $T$ is an $\alpha$-admissible mapping with respect to $\eta$;

(ii) there exists $x_{0} \in X$ such that $\alpha\left(x_{0}, T x_{0}\right) \geq \eta\left(x_{0}, T x_{0}\right)$;

(iii) $T$ is $\alpha-\eta$-continuous.

Then there exist a point $x *$ in $\overline{B\left(x_{0}, r\right)}$ such that $T x *=x *$.

Proof. Let $x_{0} \in X$ such that $\alpha\left(x_{0}, T x_{0}\right) \geq \eta\left(x_{0}, T x_{0}\right)$. For $x_{0} \in X$, we construct a sequence $\left\{x_{n}\right\}_{n=1}^{\infty}$ such that $x_{1}=T x_{0}, x_{2}=T x_{1}=T^{2} x_{0}$. Continuing this way, we have $x_{n+1}=T x_{n}=T^{n+1} x_{0}, \forall n \in \mathbb{N}$.

Since $T$ is an $\alpha$-admissible mapping with respect to $\eta$, then $\alpha\left(x_{0}, x_{1}\right)=$ $\alpha\left(x_{0}, T x_{0}\right) \geq \eta\left(x_{0}, T x_{0}\right)$. Continuing this process, we have

$$
\eta\left(x_{n-1}, T x_{n-1}\right)=\eta\left(x_{n-1}, x_{n}\right) \leq \alpha\left(x_{n-1}, x_{n}\right), \quad \forall n \in \mathbb{N} .
$$

If there exists an $n \in \mathbb{N}$ such that $d\left(x_{n}, T x_{n}\right)=0$. We assume that $x_{n} \neq$ $x_{n+1}$ with

$$
d\left(T x_{n-1}, T x_{n}\right)=d\left(x_{n}, T x_{n}\right)>0, \quad \forall n \in \mathbb{N} .
$$

First we show that $x_{n} \in \overline{B\left(x_{0}, r\right)}, \quad \forall n \in \mathbb{N}$,

$$
d\left(x_{0}, x_{1}\right)=d\left(x_{0}, T x_{0}\right) \leq r .
$$

Thus, $x_{1} \in \overline{B\left(x_{0}, r\right)}$. Suppose that $x_{2}, \ldots, x_{j} \in \overline{B\left(x_{0}, r\right)}$ for some $j \in \mathbb{N}$. Since, $T$ is an $\alpha-\eta-\psi$-GF-contraction on closed ball, such that 


$$
\begin{gathered}
G\left(d\left(x_{j-1}, T x_{j-1}\right), d\left(x_{j}, T x_{j}\right), d\left(x_{j-1}, T x_{j}\right), d\left(x_{j}, T x_{j-1}\right)\right) \\
+F\left(d\left(T x_{j-1}, T x_{j}\right)\right) \leq F\left(\psi\left(M\left(x_{j-1}, x_{j}\right)\right)\right) .
\end{gathered}
$$

This implies

$$
\begin{aligned}
& G\left(d\left(x_{j-1}, x_{j}\right), d\left(x_{j}, x_{j+1}\right), d\left(x_{j-1}, x_{j+1}\right), 0\right) \\
& \quad+F\left(d\left(x_{j}, x_{j+1}\right)\right) \leq F\left(\psi\left(M\left(x_{j-1}, x_{j}\right)\right)\right) .
\end{aligned}
$$

Since, $d\left(x_{j-1}, x_{j}\right) \cdot d\left(x_{j}, x_{j+1}\right) \cdot d\left(x_{j-1}, x_{j+1}\right) \cdot 0=0$, then there exist a $\tau>0$ such that

$$
F\left(d\left(x_{j}, x_{j+1}\right)\right)=F\left(d\left(T x_{j-1}, T x_{j}\right)\right) \leq F\left(\psi\left(M\left(x_{j-1}, x_{j}\right)\right)\right)-\tau .
$$

The rest of the proof follows from the proof of the Theorem 4 .

Along the same lines we introduce the modified $\alpha-\eta-\psi$-GF-contraction III on a closed ball.

Definition 10. Let $T$ be a self mapping in a metric space $(X, d)$ and let $x_{0}$ be an arbitrary point in $X$. Also suppose that $\alpha: X \times X \rightarrow-\infty \cup(0,+\infty) ; \eta$ : $X \times X \rightarrow \mathbb{R}_{+}$are two functions. We say that $T$ is called modified $\alpha-\eta-\psi$ GF-contraction III on a closed ball if for all $x, y \in \overline{B\left(x_{0}, r\right)} \subseteq X$; with $\eta(x, T x) \leq \alpha(x, y)$ and $d(T x, T y)>0$; we have

$$
\begin{gathered}
G(d(x, T x), d(y, T y), d(x, T y), d(y, T x)) \\
\quad+F(d(T x, T y)) \leq F\left(\psi\left(M^{\prime}(x, y)\right)\right),
\end{gathered}
$$

where

$$
\begin{aligned}
M^{\prime}(x, y) & =\max \left\{d(x, y), \frac{d(x, T y)+d(y, T x)}{2}, \frac{d\left(T^{2} x, x\right)+d\left(T^{2} x, T y\right)}{2},\right. \\
& \left.d\left(T^{2} x, T x\right), d\left(T^{2} x, y\right), d(T x, y)+d(y, T y), d\left(T^{2} x, T y\right)+d(x, T x)\right\},
\end{aligned}
$$

Moreover,

$G \in \Delta_{G}, \psi \in \Psi$, and $F \in \Delta_{F}$.

$$
\sum_{j=0}^{N} d\left(x_{j}, T x_{j}\right) \leq r, \quad \forall j \in \mathbb{N} \text { and } r>0,
$$

Now, we obtain the following generalization of Theorem 6 .

Theorem 7. Let $(X, d)$ be a complete metric space. Let $T: X \rightarrow X$ be an $\alpha-\eta-\psi-G F-$-contraction III mapping on closed ball satisfying the following assertions:

(i) $T$ is an $\alpha$-admissible mapping with respect to $\eta$;

(ii) there exists $x_{0} \in X$ such that $\alpha\left(x_{0}, T x_{0}\right) \geq \eta\left(x_{0}, T x_{0}\right)$;

(iii) $T$ is $\alpha-\eta$-continuous.

Then there exist a point $x *$ in $\overline{B\left(x_{0}, r\right)}$ such that $T x *=x *$.

Proof. The proof is same as in Theorem 6.

Remark 4. Theorem 6 and 7 generalize Theorem 3.2 of [3]. 


\section{REFERENCES}

[1] L. K. Dey, P. Kumam and T. Senapati, Fixed Point results concerning $\alpha-F$ contraction mappings in metric spaces, Applied General Topology, 1 (2019), 81-85.

[2] N. V. Dung and V. T. L. Hang, A Fixed Point Theorem for Generalized F-Contractions on Complete Metric Spaces, Vietnam Journal of Mathematics, 43 (2015), 743-753.

[3] A. Hussain, Cirić type alpha-psi F-contraction involving fixed point on a closed ball, Honam Mathematical Journal, 41 (2019), 19-34.

[4] N. Hussain and P. Salimi, Suzuki-Wardowski type fixed point theorems for $\alpha-G F$ contractions, Taiwanese Journal of Mathematics, 18 (6) (2014), 1879-1895.

[5] E. Kreyszig, Introductory Functional Analysis with Applications, John Wiley \& Sons, New York, (1989).

[6] H. Piri and P. Kumam, Some fixed point theorems concerning F-contraction in complete metric spaces, Fixed Point Theory and Applications, 214:210 (2014), 11 pages.

[7] P. Salimi, A. Latif and N. Hussain, Modified $\alpha-\psi$-contractive mappings with applications, Fixed Point Theory and Applications, 2013 (2013), Article ID: 151, 19 pages.

[8] B. Samet, C. Vetro and P. Vetro, Fixed point theorems for $\alpha-\psi$ contractive type mappings, Nonlinear Analysis, 75 (2012), 2154-2165.

[9] D. Wardowski, Fixed points of a new type of contractive mappings in complete metric spaces, Fixed Point Theory and Applications, 2012 (2012), Article ID: 94, 6 pages.

\section{Manish Chandra Singh}

Department of Mathematics

D. S. B. Campus

Kumaun University, Nainital

INDIA, 263002

E-mail address: manishnegi380@gmail.com

Mahesh Chandra Joshi

Department of Mathematics

D. S. B. CAMpus

Kumaun University, Nainital

INDIA, 263002

E-mail address: mcjoshi69@gmail.com

\section{NAVEen Chandra}

Department of Mathematics

S. N. S. Govt. PG College

Narayan Nagar, Pithoragarh

INDIA, 262550

E-mail address: cnaveen329@gmail.com 